\title{
À qui devons-nous la réédition des Relations des Jésuites?
}

\section{Thomas Charland}

Volume 3, numéro 2, septembre 1949

URI : https://id.erudit.org/iderudit/801551ar

DOI : https://doi.org/10.7202/801551ar

Aller au sommaire du numéro

Éditeur(s)

Institut d'histoire de l'Amérique française

ISSN

0035-2357 (imprimé)

1492-1383 (numérique)

Découvrir la revue

Citer cet article

Charland, T. (1949). À qui devons-nous la réédition des Relations des Jésuites? Revue d'histoire de l'Amérique française, 3(2), 210-226.

https://doi.org/10.7202/801551ar d'utilisation que vous pouvez consulter en ligne.

https://apropos.erudit.org/fr/usagers/politique-dutilisation/ 


\section{A QUI DEVONS-NOUS LA REEDITION DES RELATIONS DES JESUITES?}

Il s'agit de l'ouvrage en trois volumes, publié à Québec en 1858, sous les auspices du gouvernement canadien, chez Augustin Cốté, éditeur-imprimeur. Cette publication se présente comme le fruit de la collaboration d'un assez grand nombre de personnes. On lit, en effet, dans l'Avis de l'éditeur placé en tête de l'ouvrage:

"Bien qu'au moment de voir notre tâche accomplie, nous sentions un peu d'orgueil - sans doute légitime - se mêler au sentiment de satisfaction que nous éprouvions depuis le jour où le gouvernement canadien nous avait promis de protéger généreusement cette œuvre importante, nous croirions manquer aux devoirs de la justice et de la reconnaissance, si nous ne disions hautement combien notre travail nous a été rendu facile, grâce à l'empressement qu'ont mis les possesseurs de manuscrits et d'exemplaires de l'ancienne édition Cramoisy, à nous prêter leurs richesses. De ce nombre se trouvent $M$. l'abbé Plante, chapelain des Dames-Hospitalières de l'Hôpital-Général de Québec, qui, à force de patience et de sacrifices, a réuni la plus nombreuse collection des relations dans l'Amérique Britannique ', le Séminaire de Québec, M. l'abbé Bois, curé de Maskinongé, à l'obligeance et au dévouement duquel nous devons de posséder plusieurs manuscrits copiés par son ordre à l'étranger, et M. l'abbé Ferland, attaché à l'archevêché de Québec, qui a profité de son voyage à Paris pour nous procurer des volumes très rares... Nous n'oublierons pas non plus ce que nous devons à G.B. Faribault, qui eut la douleur de voir réduire en cendres cette bibliothèque américaine formée par lui pièce à pièce et avec tant d'amour, à M. Henri de Courcy, à M. JeanMarie Shea, historien américain, à $M$. Francis Parkman, homme de lettres de Boston, à la Bibliothèque Impériale de Paris".

1. L'abbé Plante n'en avait que vingt, au dire"de l'abbé Verreau, "Suppression des Relations de la Nouvelle-France" dans Revue de Montréal I (1877): 114. 
"A ce tribut de remercîments, nous devons ajouter ceux que méritent MM. les Abbés Plante, Ferland, et plus particulièrement M. l'abbé Laverdière, professeur au Séminaire de Québec. Pleins de dévouement pour cette entreprise que l'on peut appeler nationale, pendant des années ils ont consacré leurs loisirs à la lecture des épreuves, afin de rétablir l'ancien texte avec un scrupule qui, par respect pour les antiquaires, est allé jusqu'à reproduire fidèlement les fautes mêmes des vieilles éditions".

Disons tout de suite qu'Augustin Côté était l'imprimeur propriétaire du Journal de Québec, qu'il rédigeait conjointement avec Joseph Cauchon: ce qui explique, dans une certaine mesure, la mention particulière accordée à l'abbé Laverdière, dont le nom véritable est Cauchon. Comme s'il avait craint qu'on ne remarquât pas assez le mérite de son cousin, Joseph Cauchon écrivit, dans le Journal de Québec du 11 décembre 1858: "Après avoir reproduit l'avis de l'éditeur des Relations des Jésuites, nous avons une œuvre de justice à accomplir: l'Avis ne pouvant pas dire ce qu'on ne pouvait prévoir alors. M. l'abbé Laverdière, en corrigeant les épreuves de trois énormes volumes, avec l'intelligence, le scrupule, la patience ct l'amour d'un antiquaire, s'aperçut d'une lacune presque fatale dans un ouvrage qui contient la matière de DIX MILLE pages de l'in-douze ordinaire, et il résolut de la combler. Il manquait une table alphabétique des matières contenues dans les 42 volumes primitifs des Relations, et il la fit. Cette table, qui contient 45 pages octavo royal à doubles colonnes, est une véritable œuvre de bénédiction. Ceux qui, à l'aide de cette table, liront les Relations des Jésuites, trouvant tout comme en se jouant, ne penseront probablement pas aux veilles et au travail qu'elle a coûtés et n'en auront pas de reconnaissance à l'auteur".

"Si nous rendons cette justice tardive à $M$. l'abbé Laverdière, c'est que le premier volume qui contient l'avis de l'éditeur et le second même étaient brochés, quand ce monsieur conçut le projet de son inappréciable travail".

Or, voici que, moins de deux ans après la publication des Relations, le Dr J.-B. Meilleur écrivait, dans son Mémorial de l'éducation du Bas-Canada: ${ }^{2}$

"De tous ceux auxquels nous sommes redevables de la reproduc-

2. (Montréal, 1860), 52; seconde édition (Québec, 1876), 216. 
tion, au complet, des relations des Jésuites, Messire L. E. Bois, curé de Maskinongé, bibliophile et archéologue distingué, occupe le premier rang; car il a le mérite de l'initiative et de la plus grande somme d'efforts constants et de sacrifices généreux qu'il a fallu faire, pour procurer a l'entreprenant et courageux éditeur, les volumes et les manuscrits nécessaires à la complétion de ces relations, dont une partie a été copiée à Paris, aux frais de M. Bois. C'est par suite de sa proposition que, d'autres personnes généreuses aidant, cette tâche difficile a été entreprise et effectuée, à la grande satisfaction de nos compatriotes".

Ceux qui lisent aujourd'hui ce passage du Dr Meilleur-y compris le T.R.P. Léon Pouliot lui-même, qui le cite dans sa magnifique Etude sur les Relations des Jésuites de la Nouvelle-France ${ }^{3}$ ne soupçonnent pas ce qu'il renferme d'intentions secrètes, d'omissions calculées. Sans qu'il y paraisse, cette tranquille affirmation a un caractère belliqueux: elle entendait mettre fin à un sérieux conflit de prétentions, en même temps qu'elle voulait satisfaire une rancune personnelle, comme je vais le montrer en la replaçant dans son contexte historique.

On était à l'époque des grands collectionneurs de documents, des antiquaires, comme on disait alors. L'abbé Bois en était un des plus connus, sinon des mieux appréciés. La passion s'était allumée en lui dès ses années de collège, semble-t-il, car sa copie des Voyages de $\mathrm{Mgr}$ Plessis, écrite en partie de sa propre main, porte la date de 1833. Elle g'était sârement développée pendant son vicariat à Saínt-JeanPort-Joli (1840-43), dont le curé, son oncle François Boissonnault, lui légua une foule de précieux documents relatifs aux affaires du Chapitre de Québec. Curé de Saint-François de Beauce (1843-48) puis de Maskinongé (1848-89), il ne cessa d'enrichir sa collection de pièces de toute nature et de toute provenance.

Rien ne peint mieux notre collectionneur que cette lettre qu'il adressait à son ami Meilleur, le 11 février 1859:

"Je vous dois remercier encore une fois de l'envoi d'une lettre de feu M. Quiblier. Soyez sâr qu'elle sera sous peu relíbe au volume des autographes et qu'elle sera en bonne compagnie. Si vous en découvrez d'autres de personnages de ce calibre et qu'elles vous soient inutiles,

3. (Montréal, 1940), 33. 
disposez-en en ma faveur et tout sera bien. Une signature de P. Garnot? de Boucher-Belleville (comme auteur, gazettier, etc)? de M. Murray? de Chs. Buller? de tous ceux que vous rencontrerez. Quand donc viendrez-vous voir mes collections? Que j'ai hâte de vous exhiber mon travail de solitaire! Plans de bâtisses, cartes, plans de terrains remarquables, profils, portraits, autographes, cahiers inédits manuscrits, je réunis tout ce qui me tombe sur la main, et, sans en douter, les brochures, les journaux, les ouvrages de tous les travailleurs canadiens. Avec encore deux ou trois ans de travail et de ressources plus larges, je manquerai certainement d'espace. Depuis longtemps on me dit que ma bibliothèque est la plus considérable du clergé. C'est, diton, la plus copieuse et la plus précieuse, la plus complète, la mieux assortie. Cependant vous ne la venez pas voir. Vous avez été sourd à mes invitations depuis 7 ans! Venez donc avec vos malles de vieux et anciens pamphlets, vieux manuscrits, anciennes dissertations, etc., et apportez vos écrits et tout ce qui vous est échu de vos nombreux amis. Je vous attends toujours" 4 .

L'abbé Bois n'amassait pas les documents pour le seul plaisir de les amasser, encore moins pour la satisfaction d'être seul à les posséder. Il a généreusement ouvert ses trésors à ceux qui voulaient y puiser. La liste serait longue des chercheurs qui se sont adressés à lui par lettre ou qui sont allés le relancer dans son presbytère. Je rappellerai seulement que l'historien américain Parkman a fait à deux reprises le voyage de Maskinongé. C'est l'abbé Bois qui lui a fourni la prétendue lettre de Montcalm mourant au brigadier général Townshend; lettre qu'il n'avait pas fabriquée, comme on l'en a soupçonné, mais qu'il avait tout simplement copiée de Courrier de Québec de 1808 (III, 58).

Que des liens d'amitié se soient noués entre l'abbé et les autres collectionneurs de son espèce, la chose est toute naturelle. Qu'une petite rivalité se soit fait jour entre luj et certains de ces collectionneurs, la chose est encore toute naturelle. Ainsi je vois par une lettre du Dr Meilleur à l'abbé Bois, qu'un nommé Walker, secrétaire de la Société historique de Détroit, était venu à Montréal et que Jacques Viger l'avait accaparé pendant son séjour et ne lui avait laissé aucune chance de voir d'autres personnes. "J.V. est coutumier du fait", ajou-

4. Archives du Seminaire de Nicolet. - La correspondance Meilleur-Bois se trouve au Séminaire de Nicolet et au Scolasticat de l'Immaculée-Conception des Jésuites, à Montréal. 
tait Meilleur. "Il veut que les étrangers ne voient que lui parmi ceux qui pourraient prétendre à l'histoire ou à la chronologie". Le même jour, le 6 octobre 1858, l'abbé Bois écrivait à son ami pour lui demander copie du Journal de l'Education où se trouvait l'étude de l'abbé Verreau sur Lafiteau. "Je me proposais bien de faire ce travail moi aussi. J'ai portrait, signature autographe et notes sur le personnage, puis son ouvrage "Mœurs des Sauvages", et son mémoire sur le Ginseng, que Verreau dit n'exister que chez J. Viger et l'Hon ${ }^{\text {ble }}$ Lafontaine".

Il fait lui-même fructifier ses trésors documentaires. En plus d'innombrables communications aux journaux, notamment au Journal de Québec de son ami Augustin Côté, il a à son crédit une longue suite de livres et de brochures. Une bibliographie complète de ses écrits est pratiquement impossible à dresser, parce qu'il n'en a jamais signé un de son vrai nom. Les seuls qui portent son nom ont été publiés après sa mort: L'Isle d'Orléans, à Québec en 1895, et $M g r$ Duplessis-Mornay, dans le Bulletin des Recherches Historiques de 1912 Il en est un, pourtant, qui parut sous son nom et de son vivant: L'Angleterre et le clergé français réfugié pendant la Révolution. C'est une étude qu'il présenta à la Société Royale, en 1885. Mais, chose curieuse, la rédaction de ce morceau n'est pas son travail personnel. C'est l'abbé Thomas Hamel qui s'en était chargé pour venir en aide à son confrère devenu impotent, et qui a condensé en ces quelques pages un long chapitre que $M$. Bois avait préparé pour sa biographie de Mgr Hubert.

L'abbé Bois a touché un peu à tout. La seule inspection des titres de ses écrits révèle toutefois une prédominance des sujets de biographie ecclésiastique. J'ai montré ailleurs ${ }^{5}$ que la suprême ambition de sa vie avait été d'écrire l'histoire de l'Eglise du Canada sous forme de biographie des évêques de Québec, tout comme l'a fait après lui l'abbé Auguste Gosselin.

Les douze biographies qu'il avait terminées sont encore manuscrites, sauf celle de Mgr Duplessis-Mornay, que Mgr Henri Têtu a publiée dans le Bulletin des Recherches Historiques de 1912, "plutôt

5. L' 'euvre historique de l'abbé Louis-Edouard Bois, dans La Sociêté Canadienne d'Histoire de l'Eglise Catholique (Rapport 1935-1936): 13-24. 
comme curiosité historique que comme document", disait-il, car "la biographie de Mgr de Mornay est remplie d'erreurs", et "l'abbe Bois a fait un panégyrique" ${ }^{6}$.

Je viens de toucher aux deux principaux défauts qui entachent toutes les productions historiques de l'abbé Bois et qui lui ont valu, de son vivant même, une bien mauvaise réputation auprès des érudits, et en particulier de Jacques Viger. Ce dernier avait la phobie des erreurs de date et d'orthographe. Elles lui déplaisaient à ce point qu'il englobait dans sa réprobation la personne même des auteurs et jusqu'a leurs amis. Et sa haine était tenace, comme nous allons le voir à propos de l'abbé Bois.

Un des premiers écrits de l'abbé fut son Esquisse de la vie et des travaux apostoliques de Sa Grandeur Mgr Fr. Xavier de Laval-Montmorency, premier évêque de Québec, suivie de l'éloge du Prélat. Cette brochure porte la date de 1845; mais elle ne fut distribuée qu'en décembre 1846, l'impression en ayant été retardée par la catastrophe du 28 juin $1845^{7}$, à savoir l'incendie des faubourgs St-Jean et St-Louis à Québec. Faribault en envoya un exemplaire à Jacques Viger pour ses étrennes, en lui demandant ce qu'il en pensait. Viger se montra impitoyable pour l'aspirant auteur. "Le fond de l'ouvrage est bun", disait-il, "c'est une justification inoffensive et complète de la conduite de l'Évêque et qui réfléchit sévèrement sur la manière de penser de M. G[ arneau ] et sur sa manière de juger le saint Évêque... Du reste, cette Esquisse ne m'apprend pas grand chose en fait de particularités sur la vie de Mgr de Laval; je savais à peu de chose près tout ce q' '’il nous dit, mais elle profitera à d'autres et elle ferait honneur au nom canadien si elle abondait moins en anglicismes (et par cela ne décelait une plume moderne et du pays), et si elle ne renfermait point de graves et nombreuses inexactitudes que bien peu de personnes au pays (peutêtre 2 ou 3) sauront relever, et qui vont rester et se perpétuer par la répétiti in". Bref, s.uus ce rapport, "un mauvais livre de plus lancé dans la société" 8 .

Viger voulait que Faribault montrât sa lettre à Ronald McDonald et le priât d'en tirer un ou deux articles pour son journal, le Canadien.

6. BRH, XVIII (1912): 319 note.

7. Journal de Québec, 9 mars 1847.

8. Viger a Faribault, 8 février 1847 (Archives de la Province de Québéc). 
"S'il juge à propos de les présenter comme articles éditoriaux", ajoutait-il, "qu'il se les adresse comme articles communiqués venant de Montréal, s'il veut, et signés Nicolas... Si Mr McDonald fait ce que je suggère, corrigez les épreuves, je vous prie, afin qu'il n'y git pas de fautes typographiques dans mes corrections surtout; sans quoi, vous concevez quelle confusion, etc. résulterait de ces incorrections et comme on tomberait sur le correcteur-Nicolas. Mes chiffres, mon orthographe doivent être rendus rigidement et sans faute aucune; ou bien... plus de mal que jamais. Vuuz m'entendez, j'en suis sûr. Sat est'.

Ce qu'il suggérait fut fait. Le Canadien de 24 février 1847 contenait un "Extrait de la lettre de Nicolas à son ami Alexis, au sujet de la brochure intitulée: Esquisse..." M. Bois en fut atterré. Il se croyait en bons termes avec Viger, et il avait été prévenu par le Dr Meilleur que le "grand vicaire" montréalais était très content de sa brochure. Il eat voulu que son ami allât voir Viger; mais Meilleur crut plus prudent de n'en rien faire: "Il est un peu furet et moi naif, et je crains qu'une entrevue ne servirait qu'à nous compromettre vous et moi" '

Des journaux tentèrent de réfuter les critiques de Viger, ou du moins d'en atténuer la portée. Et l'abbé se résigna à son sort, tout ex s'obstinant à écrire sous l'anonymat. Viger n'arriva jamais à l'estimer, loin de là. N'écrivait-il pas à Faribault, le 19 décembre 1856: "J'ai fait venir de chez $M$. Côté dernièrement quatre petites brochures pour moi et quelques amis. Ce sont encore des productions du fameux Lignum... Tous ces would be authors sont vraiment à tuer à coup d'épingles et leurs imprimeurs, pour faire leurs pâques, auront à se confesser de leurs petits larcins de 15 sols par brochure. Quelques amis prêtres m'ont demandé un nouveau Nicolas à son ami Alexis. Je n'en ferai rien pour le moment... Je n'entends point comprendre Mr Ferland dans ce ramassis d'écrivains canadiens: il a donné, lui, des preuves respectables de son talent, de sa capacité et surtout de son honnêteté, il n'est ni pilleur, ni menteur, ni incorrigible comme G[ eorges ] L[ emoine ] et B[ ois ]. Quelle honte pour le pays!"'

En 1857, l'abbé Bois réditait l'État présent de l'Église et de la Colonie française dans la Nouvelle-France par M. l'Evêque de Québec, en le faisant précéder d'une courte notice de Mgr de Saint-Vallier. Nouvel accès de colère chez Viger. Le Dr Meilleur apprend à l'abbé

9. Meillour a Bois, 12 mars 1847 (Archives du Stminaire de Nicolet). 
Bois (19 septembre 1857): "Bibaud a encouru l'ire de Jacques, parce qu'il a pris pour vous, et qu'il a dit qu'il ne valait pas la peine de faire tant de tapage pour une petite erreur de date, que le monopole n'est pas du ressort exclusif de J... Il est certain que J. ne veut pas souffrir que les autres se mêlent d'histoire et surtout de chronologie à l'égard du Canada".

L'année suivante, dans son Panthéon Canadien, Maximilien Bibaud fit preuve d'un zèle indiscret en faveur de l'ami Bois. Pour comble de maladresse, il exaltait ses mérites au détriment de ceux de Jacques Viger, car c'est bien ce dernier qui me semble visé dans le passage suivant: "Les notices biographiques des évêques de Québec, Laval et St-Vallier, du commandeur de Sillery et du docteur Sarrasin, et l'Eloge de Montcalm, avec l'État de l'Église et la collection complète des Relations des Jésuites, à laquelle il a eu une grande part, forment déjà, en dépit de quelques erreurs, une collection plus variée, plus large et autrement importante que les fragments archéologiques mis à jour en Canada avant lui. Il aura le mérite d'avoir fourni beaucoup de données pour le Panthéon canadien".

Tout ce qui précède nous aide à comprendre les appréhensions qu'éprouvait le pauvre abbé Bois au moment où la réédition des Relations allait être lancée dans le public, et qu'il confiait au $\mathrm{Dr}$ Meilleur, dans sa lettre du 6 octobre 1858:

"Ne vous ennuyez pas trop des Relations qui m'ont assez ennuyé maintenant et sur lesquelles j'ai blanchi - comme aussi pour lesquelles j'ai blanchi. Vraiment ce travail m'a affaibli, m'a tracassé. Mon cher Evêque n'en paraît pas trop édifié et mon voisin me rapporte du saint homme des propos peu encourageants que j'ai raison de croire véridiques puisqu'il m'a lui-même honoré de l'expression (non équivoque) de ses sentiments. Néanmoins j'avais eu l'âme assez bonne que de croire que le tout n'avait pas été au delà de nos deux individualités. Cependant le cher Supérieur en a jugé autrement. Non que ce m'afflige beaucoup, mais son mécontentement me fait quelque peine. Et je n'en voudrais faire à personne. Voyez, déjà avant que l'œuvre soit au jour, qu'on l'ait flairee, on y trouve a redire! Que sera-ce quand la chose aura été distribuée, quand les messieurs de la presse braqueront leurs lunettes sur les 3 volumes!

Quid sum miser tunc dicturus, Quem patronum rogaturus? 
Mais je tâcherai d'être impassible et de ne pas voir les légitimes colères qu'aura provoquées encore une fois ma témérité. Encore si j'avais réputation, renom, un nom qui décidât au respect les plus osés, on me pardonnerait cette reproduction de 40 vols. ou plutôt de 45 en faveur du motif qui m'animait! Je désirais mettre entre les mains de ceux qui voudront travailler sur l'histoire des documents sârs et tout trouves. J'ai passé mes meilleures annćes à colliger, à transcrire, à exhumer des matériaux et je vais par là offenser ceux qui avaient aussi réuni de ces antiques volumes et qui les possédaient pour dire: Je les ai et je suis le seul qui les ai. - Je sais qu'il y a là des documents qui, avant que je les eusse fait transcrire en Europe, n'avaient jamais traversé l'océan. Jugez des aigreurs qu'on va me réserver et des gronderies que j'ai provoquées! Voilà bien le moment pour moi de chercher un souterrain".

"Mais c'est trop laisser liberté à ma plume. Je me hâte de vous dire que je suis grandement redevable à Mr l'abbé Laverdière, prêtre du Séminaire de Québec, pour sa collaboration: il a corrigé presque toutes les épreuves et fait bonne partie des tables. J'oubliais de vous dire que le vrai nom de cet abbé est Cochon et qu'il est le cousin de l'Hon ${ }^{\text {ble }}$ Cauchon. Par obséquiosité et par reconnaissance, le nom de Mr Ferland se trouve aller avec le sien, car ce bon monsieur nous a fait copier en France des volumes que nous n'avons pas pu acheter il nous a aussi acheté (à notre compte, s'entend) quelques-uns des volumes qui ne se trouvaient pas en Canada - mais les plus rares Relations de 1611, de 1626, de 1632 - 1633, m'appartenaient et manuscrites quand j'ai mis la chose en jeu - j'en avais d'autres volumes imprimés. Et voilà. - Dans quelques jours mes manuscrits me reviendront".

A la fin de septembre tout était prêt, sauf les tables. La distribution ne devait se faire qu'après que le gouvernement aurait reçu sa part - 1000 exemplaires - et compté ses deniers. Les curieux se lamentaient. Jacques Viger était au désespoir de ne point les recevoir. Eut-il le temps d'y jeter un coup d'œil, et de constater qu'il n'était point fait mention de lui dans l'Avis de l'éditeur ?. ${ }^{10}$ Toujours est-il

10. Viger avait fait un travail sur la collection des Relations que possédait la Bibliothèque du Parlement à Québec avant l'incendie de février 1854: "Liste des Titres de chacun des volumes de la collection entière des Relations publiées de 1632 a 1672 , etc., etc., avec notes par J.V." 
que, le 12 décembre, il succombait à une hydropisie du péricarde. L'abbé Bois dut éprouver un gros soulagement en apprenant la mort de l'impitoyable censeur. Mais il n'était pas au terme de ses tribulations. Au moment où Viger expirait, une polémique venait de s'engager dans les journaux à propos de l'initiative de la réédition. Déjà, on avait pu lire, dans le numéro du 19 novembre du Courrier du Cana$d a$, rédigé par J.-C. Taché et tout dévoué à Chauveau:

"Comme il convient de donner à chacun la part qu'il a dans la chose, nous devons dire que c'est à l'honorable $M$. Chauveau que le pays doit l'idée de cette ré-impression de ces mémoires importants, qui deviennent de plus en plus rares et dont plusieurs ont déjà disparu complètement du commerce de la librairie. Nous trouvons, en effet, dans le Journal de l'Assemblée Législative, session de 1854-55, séance du 2 octobre, la résolution et l'ordre suivants: "Sur motion de l'Honorable M. Chauveau, secondé par l'honorable M. Chabot, Résolu: Qu'il soit présenté une humble Adresse à Son Excellence le Gouverneur-Général, priant son Excellence de faire imprimer, en sus des Documents mentionnés dans une Adresse de cette Chambre, les Documents qui ont été obtenus et extraits des Archives Publiques, a Paris et à Londres, et qui sont maintenant en manuscrit dans la Bibliothèque du Parlement, ou dans la Bibliothèque de la Société Littéraire et Historique de Québec, ou qu'on pourra se procurer par la suite, et qui paraîtront d'un intérêt suffisant au point de vue légal et historique; et aussi, de faire ré-imprimer ceux des ouvrages publiés dans les premiers temps de la Colonie, qui peuvent être d'une grande valeur et sont devenus très rares, les dits ouvrages et Documents devant être imprimés dans la forme et avec les notes et cartes qui seront jugés convenables, et assurer son Excellence que cette Chambre s'empressera de subvenir aux dépenses nécessaires pour l'accomplissement des objets susdits.

"Ordonné, que la dite Adresse soit présentée à Son Excellence le Gouverneur-Général, etc. etc."

Cette réclamation étant restée sans écho, le Courrier du Canada revenait à la charge, le 10 décembre suivant, après avoir reproduit l'Avis de l'éditeur des Relations:

"Nous sommes heureux de voir mentionner dans cet avis les noms si chers à notre histoire de MM. Plante, Bois, Ferland, Faribault, de Courcy et Laverdière et encore ceux de MM. Shea et Parkman. 
Comme nous voulons donner à l'Editeur M. Cóté toute sa part de mérite, nous ajouterons, à ce que nous avons déjà dit de cette publication, un détail qui l'honore et que nous tenons de bonne source. M. Côté a, de bonne grâce et à ses propres dépens, ajouté à la collection, sur la demande d'amis de notre histoire, deux relations que, par son traité avec le gouvernement, il n'était point tenu d'imprimer:- le public doit lui tenir compte de ce sacrifice".

"La part faite à l'éloge mérité, il nous est impossible de ne pas formuler contre l'Editeur un reproche très grave; car il ne s'agit pas d'une publication ordinaire et sans grande valeur; mais d'une œuvre nationale. M. Côté a omis de faire mention de la résolution, adoptée par la Chambre d'Assemblée, sur la proposition de M. Chauveau, en vertu de laquelle cette publication a été faite aux frais du gouvernement. Au contraire M. Côté donne expressément à entendre qu'il a entrepris ce trâvail à ses risques et périls et par pur patriotisme; la seule mention qu'il daigne faire du gouvernement canadien, qui le paie largement pour cette entreprise, se trouve dans les quelques mots suivants insérés sur la page titre: ouvrage publié sous les auspices du gouvernement canadien et dans quelques mots vagues et sans signification de l'avis: or ceci ne dit pas du tout que c'est aux frais du gouvernement canadien, que cet ouvrage a été publié, - et une pareille omission n'est pas excusable. Il n'est pas juste d'avoir passé sous silence la part qu'ont dans cette publication la Chambre Législative du Canada et MM. Chauveau et Chabot qui ont été les moteur et secondeur de la proposition. Quoi, dans cet ouvrage que l'Etat a fait imprimer à beaux deniers, il n'y a pas un mot qui fasse clairement mention du fait! Loin de là, l'éditeur, au mépris de la justice, des usages et des convenances, vient nous dire:" C'est à la sollicitation d'un grand nombre d'entre eux (les amis de notre histoire) que nous avons entrepris de publier une nouvelle édition d'un ouvrage aussi considérable". Et la sollicitation du gouvernement, faite sous forme d'une couple de mille louis, n'y est-elle pour rien? Mais c'est quelque chose d'incroyable! M. Côté a été mal inspiré cette fois et, en refusant justice à qui elle est due, il se fait plus de tort à lui-même qu'aux autres".

Le Journal de Québec et le Courrier du Canada étaient à couteaux tirés depuis quelque temps. Provoqué directement, cette fois, le Journal de Québec infligea à son antagoniste la cinglante mise au point que voici: 
"La haine du Courrier du Canada perce partout. Hier, il adresse des reproches amers à M. Côté au sujet de la publication des Relations des Jésuites. Il lui fait un crime de n'avoir pas dans l'avis de l'éditeur fait mention de la résolution adoptée par la chambre d'assemblée en 1854, sur la proposition de $M$. Chauveau, en vertu de laquelle, dit-il, cette publication a été faite aux frais du gouvernement du Canada; il l'accuse encore de n'avoir pas assez dit, dans le titre et l'avis, la part prise à cette publication par le gouvernement".

"Ces accusations nous obligent nécessairement de mettre les choses à leur place et de donner à chacun sa part de mérite".

"En 1853, le Rév. M. Bois, curé de Maskinongé, poussait M. Cóté à entreprendre la publication des Relations des Jésuites, et offrait de donner $£ 100$ et même plus, s'il le fallait, pour sa part d'aide, de contribution et de sacrifices. La résolution dont parle le Courrier n'existait pas alors: la première idée de la publication appartient donc a M. le curé Bois. C'est après cela que des amis de l'entreprise s'adressèrent à l'honorable M. Morin, alors ministre, qui promit au nom du gouvernement une part de coopération; c'est parce que M. Morin avait promis que ses successeurs, et notamment M. Cartier, alors secrétaire provincial, donnèrent l'encouragement libéral qui a si puissamment aidé au succès de la publication canadienne jusqu'ici la plus importante".

"C'est a la suite de la promesse de M. Morin, que M. Chauveau proposa la résolution d'où sont sorties tant de publications, parmi lesquelles celle des Edits et Ordonnances. Cette résolution ne mentionne en aucune manière les Relations des Jésuites, et les eut-elle mentionnf́es nominalement que l'éditeur ne pouvait pas être taxé d'injustice en omettant d'en parler dans son avis, car $M$. Chauveau agissait, en cette occasion, comme membre du gouvernement, et il suffisait de donner au gouvernement sa part de juste mérite. M. Côté l'a fait dans le titre et dans l'avis".

"Mais nous ne doutons pas que M. Chauveau et M. Chabot eussent en vue les Relations des Jésuites en proposant leur résolution de 1854, et si l'éditeur eât été éclairé sur leur pensée il n'eât pas hésité à leur donner leur part de mérite dans cette cuvre patriotique".

"Après cela, il n'est pas vrai que la publication entière ait été faite aux frais du gouvernement, bien que nous soyons prêt à admettre que, sans le secours très libéral du gouvernement de 1856, il eat été, malgré 
le concours de quelques particuliers, peut-être impossible à M. Côté d'entreprendre cette publication".

"Le gouvernement s'est engagé à prendre mille exemplaires des Relations, si M. Côté les publiait, et ces mille exemplaires lui ont été délivrés. En cela le gouvernement a fait ce qu'il fait pour toutes les publications importantes. C'est ainsi qu'on a pris un certain nombre d'exemplaires de l'histoire du Canada de M. Christie et des deux premières éditions de l'histoire de Garneau, et que l'on s'est engagé à en prendre un certain nombre aussi de la troisième édition du même et important ouvrage. Et pour tout cela on n'a pas attaque MM. Christie et Garneau, parce qu'ils n'ont pas mis dans le titre et la préface de leurs livres: "jmprimé aux frais du gouvernement".

"Les Relations des Jésuites étaient plus considérables, le gouvernement donnait davantage, donnait assez pour rendre la publication possible; aussi M. Côté a-t-il plus dit que ces écrivains. Il a dit au frontispice de cet ouvrage, colossal pour le Canada, qu'il était publié sous les auspices du gouvernement; il a même dit plus que cela; car il a écrit ces lignes dans l'avis de l'éditeur trouvé si vague par le Courrier:

"Bien qu'au moment de voir notre tâche accomplie, nous sentions un peu d'orgueil, sans doute légitime, se mêler au sentiment de satisfaction que nous éprouvions depuis le jour où le gouvernement canadien nous avait promis de protéger généreusement cette auvre importante, nous croirions, etc."

"Le Courrier est bien au delà de la vérité quand il parle de deux mille louis".

Le Courrier dut encaisser le coup, et il se consola en répliquant (13 décembre) que l'entreprise était une excellente spéculation pour l'éditeur ${ }^{11}$. Mais l'affaire n'en resta pas là. Dans sa livraison de décembre 1858, le Journal de l'instruction publique eut soin de reproduire du Courrier du Canada, ce qui y était dit de l'initiative de Chauveau dans la réédition des Relations. Le Dr Meilleur écrivit à l'abbé Bois: "Je mettrais ma main dans le feu en preuve de la conviction où je suis que Ch. est l'auteur de l'article du Courrier! et qu'il reproduit comme il fait de ses autres articles qu'il envoie tout faits aux journaux* et qu'il reproduit ensuite comme venant d'eux. Taché est son instru-

11. Le Gouvernement y affecta la somme de $£ 1400$. Cf. Pouliot S.J., Etudes sur les Relations, 32. 
ment dévoué, aussi ne vous y fiez pas plus qu'il ne faut. Je vous parle comme vous me permettez de le dire: en ami". Et il ajoutait: "Cependant, je regrette infiniment de voir comme vous êtes maltraité, et ce par vos amis, Côté et Cauchon. Celui-ci devrait voir à ce que celui-là vous rendit justice. Si je puis faire quelque chose, ce sera de tout cœur, mais, véritablement, je ne sais pas écrire, et puis, je suis complètement absorbé par un embarras pécuniaire ou plutôt par les cruelles inquiétudes qu'il me cause" 12 .

Le Dr Meilleur approcha un journaliste de Montréal, Joseph Royal, de l'Ordre, à qui il donna les informations voulues au sujet des Relations. Royal promit de faire un article, mais ne s'exécuta pas, par crainte de déplaire à Chauveau. Sur quoi Meilleur écrivit à l'abbé Bois: "Ce sont tous gens à servir d'instrument à Ch. qui, paraissant une puissance, a auprès d'eux l'influence qu'a toujours un homme qui la possède auprès des ambitieux et des nécessiteux... Ainsi, j'en reviens à ma première idée: c'est que les journaux d'ici, qui sont sous la patte de Ch., ne vous serviront pas" 13.

Ayant échoué auprès des journaux, Meilleur voulut profiter d'une autre occasjon qui se présenta de faire rendre justice à son ami. L'imprimeur John Lovell devait publier une traduction anglaise des Relations, qu'il avait demandée au Dr William Wilson, traducteur à la Chambre d'Assemblée ${ }^{14}$. Meilleur alla voir Lovell. Celui-ci, convaincu du bon droit de l'abbé Bois, mais ne voulant pas "se mettre aux prises avec d'autres", demande "un renseignement exact mais laconique", qu'il reproduirait in extenso. L'abbé Bois rédigea donc une note, que le Dr Meilleur fit traduire par son fils Alphonse. Lovell la remit au Dr Wilson, en lui recommandant d'en faire usage comme bon lui semblerait dans l'intérêt de la chose. Lovell comptait sur un bon nombre de souscriptions, et surtout sur une subvention du gouvernement. En août 1860, la traduction du premier volume était prête; mais l'imprimeur n'avait encore rien obtenu du gouvernement. Ce dernier jugea-t-il qu'il avait déjà assez dépensé pour l'édition fran-

12. Meilleur à Bois, 21 déc. 1858 (Archives du Scolasticat de l'ImmaculéeConception).

13. Le même au même, 21 déc. 1858 et 3 janv. 1859.

14. A la Bibliothèque de la Ville de Montréal, collection Gagnon, se trouve une lettre de A.-N. Morin à l'abbé Ferland (14 oct. 1859) pour lui présenter Wilson. Cf. Philéas Gagnon, Essai de Bibliographie Canadienne, I (Québec 1895): 414. 
çaise? Y eut-il intervention de la part des autres prétendants, suxquels la note relative à l'abbé Bois n'aurait pas plu? Toujours est-il que le projet de Lovell n'eut pas de suite. S'il avait été exécuté, nous n'aurions peut-être pas la splendide Gdition de Thwaites, The Jesuit Relations and Allied Documents (Cleveland, 1896-1901).

Vers le même temps parut le Mémorial de l'éducation du BasCanada ${ }^{15}$. Grand émoi au Bureau de l'Education et à la Socióté historique de Montréal. Le Dr Meilleur se mêlait d'écrire sur un sujet dont Chauveau devait avoir le monopole. Bien plus, il ne disait pas un mot de Chauveau, dans le passage relatif aux Relations que j'ai cité au début de cet article. Il en avait prévenu son ami Bois (19 février 1859): "Soyez certain que je n'attribuerai pas à Ch. le mérite de la reproduction des relations des Jésuites, mais, comme de raison, je dois ne pas m'immiscer dans la discussion à ce sujet. Ch. trouvera assez de quoi lui faire faire la grimace sans cela dans le Mémorial".

Il ne disait pas un mot non plus - et ceci parait plus étrange de la subvention accordée par le gouvernement à l'éditeur-imprimeur. Il écrivait tout simplement: "C'est par suite de sa proposition (l'abb6 Bois) que, d'autres personnes généreuses aidant, cette tâche difficile a été entreprise et effectúé, à la grande satisfaction de nos compatriotes". C'est que le Dr Meilleur en avait contre le gouvernement, comme il en avait contre Chauveau. Tout le temps qu'il avait été surintendant de l'instruction publique pour le Bas-Canada (1842-1855), il avait lutté pour empêcher que son département servit d'instrument politique. On lui avait fait payer cher son indépendance. Son salaire était à peine suffisant pour faire vivre sa nombreuse famille. La pression étant devenue trop forte, il avait démissionné pour faire place a un compétiteur, auteur d'un "roman assez insignifiant"16. Sa correspondance avec l'abbé Bois est remplie de jérémiades au sujet de la mesquinerie du Gouvernement a son endroit, de remarques satiriques sur les moindres faits et gestes de son successeur, particulièrement sur le faste dont il s'entourait: une demi-douzaine de plumes d'ivoire montées en argent, de quatre piastres et demie chacune, des tapis pour une valeur de $£ 100$, un des plus beaux carrosses de la ville, tiré

15. Il fut annoncé par un article de Cyrille Boucher, dans l'Ordre du 15 aodt 1860.

16. Meilleur a Bois, 10 oct. 1856 (Archives du Séminaire de Nicolet). - Il s'agit de Charles Gutrin par P.J.O. Chauveau (Montréal, 1853). 
à deux chevaux, etc. Il eut même une discussion publique avec Chauveau, qu'il ridiculisa pour avoir désigné par le mot sarrasine (au lieu de sarracène) la plante découverte par le Dr Sarrazin.

Le Dr Meilleur n'eut pas le dernier mot au sujet de la réédition des Relations. L'année qui précéda celle de sa mort, il put lire, dans la Revue de Montréal, un article sur la Suppression des Relations de la Nouvelle-France, où l'abbé Verreau disait: "Les éditions originales des Relations auront toujours une valeur intrinsèque, que les plus belles impressions ne peuvent leur enlever. Celle que notre gouvernement a fait exécuter à Québec, en 1858, est loin de réunir les conditions de beauté et de commodité qu'on pouvait exiger pour le monument élevé à notre histoire. Le mérite - et il n'est pas médiocre - de cette édition, c'est d'avoir réuni définitivement et mis à la portée de tous les hommes d'étude, des volumes dont il est si difficile de dresser même une liste complète et exacte. C'est l'Hon. M. Chauveau qui décida le Gouvernement à les sauver d'une entière destruction, et cette seule mesure, qu'il ne put mettre à exécution lui-même, suffirait pour lui assurer la reconnaissance des esprits cultivés" ${ }^{17}$.

Et voici le dernier épisode de cette lutte entre la vérité et la vanité. En 1883, paraissait le premier volume de la Collection de manuscrits relatifs à la Nouvelle-France, éditée sous les auspices de la Législature de Québec. C'est encore l'abbé Bois qui était au fond de l'entreprise. C'est lui qui avait suggéré cette publication à l'Honorable Jean Blanchet, son ancien protégé devenu secrétaire de la Province, qui en avait fourni les matériaux, corrigé les épreuves et rédigé l'introduction. Or, on lit dans cette dernière (p. VI): "La seule entreprise d'un intérêt historique faite jusqu'à ce jour, par le gouvernement de ce pays, est la publication des É dits et Ordonnances en 1803, réédités d'une manière beaucoup plus ample et plus complète vers 1856". Pourquoi parler de cette publication comme de la "seule entreprise... faite jusqu'à ce jour par le gouvernement", sinon pour exclure la participation directe du Gouvernement à la réédition des Relations des Jésuites en 1858 ? Je ne crois pas me tromper en affirmant que telle était l'intention du rédacteur de cette assertion apparemment anodine. Et je ne crois pas me tromper non plus en voyant une riposte dans ce qu'écrivit Chauveau, deux ans plus tard, dans l'Introduction des

17. Revue de Montréal I (1877): 116. 
Jugements et délibérations du Conseil Souverain de la Nouvelle-France (I, p. VI.): "Le 2 octobre 1854, j'eus l'honneur, étant secrétaire de la Province dans l'administration MacNab-Morin, d'obtenir de la législature la permission de faire imprimer aux frais du gouvernement les documents historiques et les anciens ouvrages devenus rares; cette proposition ne fút exécutée que sous l'administration suivante, et la publication des Relations des Jésuites en fut le premier résultat". Après s'être ainsi attribué le premier rang, Chauveau daignait accorder à son compétiteur cette fiche de consolation: “M. Bois, qui s'était procuré à grands frais un bon nombre des volumes les plus rares de cette précieuse collection, et $\mathrm{M}$. Laverdière, qui nous a donné plus tard une si belle édition des Oeuvres de Champlain, dirigèrent cette réimpression". Et ainsi se termina, au détriment de la vérité, un des plus longs conflits des annales de l'édition canadienne.

L'abbé Bois ne mourut pas sans avoir vu ses mérites d'historien couronnés. En 1882, le Gouverneur Général l'avait invité à faire partie de la Société Royale, qu'il venait de fonder. En 1883, l'Université Laval lui avait remis un diplôme de docteur ès lettres honoris causa. En 1885, Mgr LaFlèche l'avajt créé chanoine honoraire de sa cathédrale des Trois-Rivières. Mais je soupçonne que, en dépit de sa grande modestie, il aurait préféré à tous ces honneurs la reconnaissance de ses droits. Serait-il satisfait de savoir que je les aj revendiqués à la tribune jadis occupée par Jacques Viger et l'abbé Verreau ${ }^{18}$ ?

Thomas Charland, O.P.

18. A la Société historique de Montréal. 